\title{
Multiple sclerosis and
}

\section{eye: Geometric complexity of the optic nerve head}

Received: 13 January, 2020

Accepted: 05 February, 2020

Published: 07 February, 2020

*Corresponding author: G Bianciardi, Department of Medicine, Surgery and Neuroscience, University of Siena, Italy, E-mail: giorgio.bianciardi@unisi.it

https://www. peertechz.com

\section{microvascularity-a pilot study}

Check for updates

\section{G Bianciardi* and C Traversi}

Department of Medicine, Surgery and Neuroscience, University of Siena, Italy

\begin{abstract}
Multiple Sclerosis (MS) is a progressive neurodegenerative disease of the central nervous system characterized by focal demyelinating lesions in the white matter of the brain or spinal cord. When MS affects the nerves in a person's eyes, it can cause Optic Neuritis (ON). We have investigated the microvascular pattern of the nerve head performing the evaluation of its fractal dimension (geometric complexity). Mean geometric complexity of the optic nerve head microvascularity in the affected eye in patients with multiple sclerosis resulted higher than in control normal subjects $(p<0.01)$, and, moreover, the unaffected eye in patients with multiple sclerosis showed a geometric complexity that was not significantly different from the one of the affected eye and higher than in control normal subjects $(p<0.01)$. These data, if confirmed in a higher number of patients, could show a characteristic microvascular pattern of the optic nerve head in subjects affected by multiple sclerosis, giving us a possible tool to identify earlier subjects that can develop multiple sclerosis.
\end{abstract}

\section{Introduction}

Multiple Sclerosis (MS) is a progressive neurodegenerative disease of the central nervous system characterized by focal demyelinating lesions in the white matter of the brain or spinal cord. Lesions cause a range of vary symptoms depending on the location of lesions, and can include changes in autonomic, motor or sensory functions $[1,2]$. In particular, for the purpose of this article, MS often presents early ocular manifestations secondary to inflammatory demyelination of the visual pathway, also causing optic neuritis [3]. We can recall that a majority of patients experience at least one episode of ocular involvement in the course of their disease $[4,5]$.

Fractal analysis is emerging as a powerful tool to perform differential diagnosis and prognosis of the patients in pathology as well to improve the effectiveness and safety of patient care [6-8]. A lot of papers focused the usefulness of fractal analysis in the study of the retinal vessels [9-14]. In this paper we present a study of the microvascular pattern of eyes in MS patients, studying by fluorangiography and fractal analysis the optic nerve head microvascularity.

\section{Materials and methods}

\section{Patients}

Patients with unilateral optic neuritis without optic disc oedema $(\mathrm{ON})$ affected by MS $(n=6)$ were recruited from the Department of Medicine, Surgery and Neuroscience at the University of Siena. Three patients were female and three were male (mean age $=32.3$ years). In the control group, six patients were female and seven were male (mean age $=48.7$ years).

Each patient underwent a complete neuro-ophthalmic examination, including assessment of visual acuity, colour vision, papillary reaction, slit-lamp examination, applanation tonometry, Goldman visual field testing, visual evoked potentials, dilated fundoscopy and fluorescein angiogram. All patients were examined using a fluorescein angiogram within the first two weeks after they reported the first symptoms and before treatments; all patients gave informed consent. Fluorescein angiogram of normal subjects $(n=13)$ were used as controls. The protocol for this research project was approved by the Ethics Committee of the University of Siena and it conforms to the provisions of the Declaration of Helsinki, 1955 (as revised, Edinburgh, 2000). 


\section{Image analysis}

Static fluorescein angiogram was performed (IMAGEnet 2000,v.2.0, Topcon). The early, intermediate and late phases of the angiogram were studied. Images of the early venous phase of the angiogram (around 20 seconds) gave the best visualization of the optic disc vessels and were saved (Figure 1). The same magnification was used for each subject.

A manual outline of the trajectories of the two-dimensional microvascular network was performed down to microvessels of 20 micron of diameter, processed to threshold the vessel network without background interference and converted into an outline of one pixel (Figure 2) by means of Jmicrovision, http://www.jmicrovision.com and Image Analyzer softwares http://www.fosshub.com/Image-Analyzer.html .

\section{Geometric complexity, D0}

Briefly, the skeletonized image is covered by nets of square boxes, from 10 pixel to 130 pixels wide and the amount of boxes containing any part of the outline is counted. A log-log graph is plotted on the side length of the square against the number of outline-containing squares. If our image is fractal, a logarithmic linear segment appears: the slope of the linear segment of the graph represents the local fractal dimension [15] of the image (Figure 3). The correlation coefficient of the straight line was always above a value equal or greater than 0.99, thus justifying our fractal approach. The methodology was implemented using Benoit 1.3 software (TruSoft Int'l Inc, http://trusoft-international.com/.html) and validated measuring computer generated Euclidean and fractal shapes of known geometric complexity. Inter-and intra-observers errors were $<3 \%$.

\section{Statistical analysis}

Mann-Whitney's U test was used in order to compare the fractal indexes between the groups and to ascertain the statistical significance; regression analysis was used to ascertain the log-log plot linearity.

\section{Results}

Geometric complexity of the optic nerve head

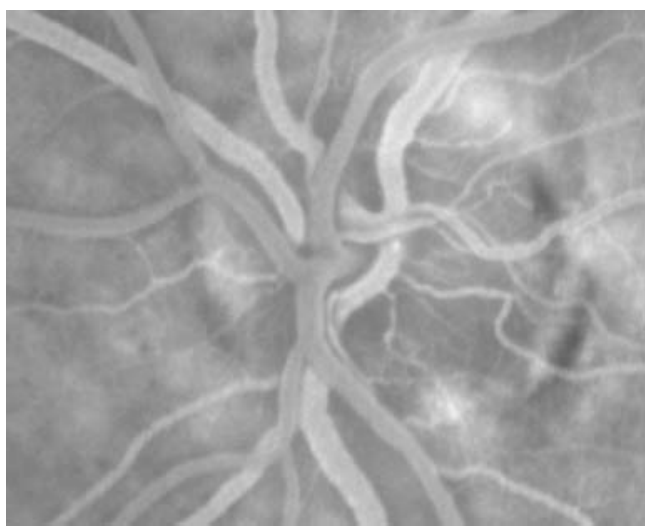

Figure 1: Fluorescein angiography. Microvascular pattern of the optic nerve head in a normal subject.

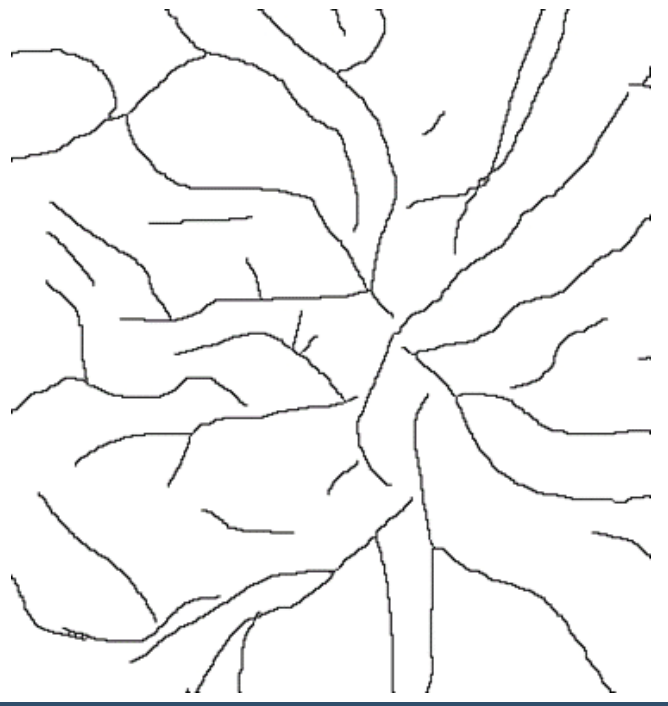

Figure 2: Trajectories of the optic nerve head microvascularity in a normal subject.

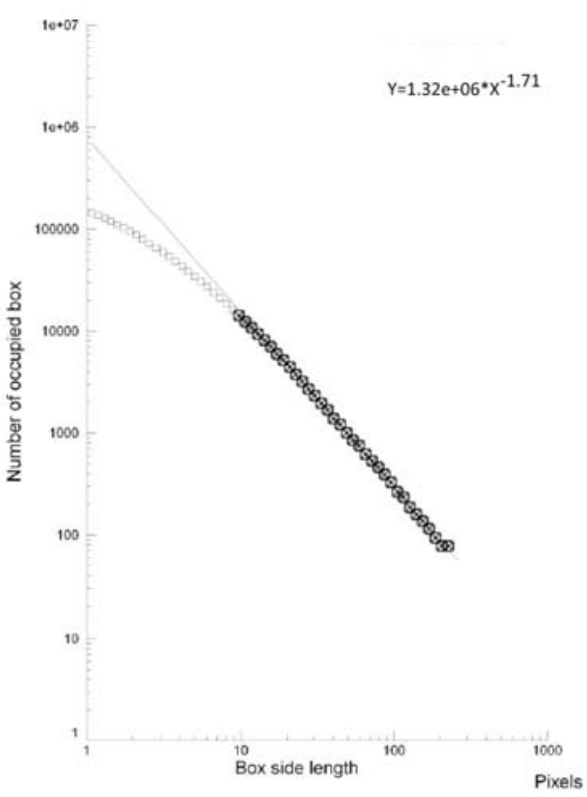

Figure 3: Log-log plot of the trajectories of the optic nerve head microvascularity. The feature is fractal (a straight line is present in the log-log plot: the structure is statistically self-similar), its slope is the fractal dimension (D0) at the chosen scales. Benoit 1.3 software.

microvascularity (affected eye) in MS patients showed a higher value than in normal subjects (Figure 4, Table 1).

Geometric complexity in unaffected eyes of the MS patients was not significantly different from that of the affected eye (Figure 5, Table 2). Both the eyes, affected and unaffected one, revealing a geometric complexity higher than in normal control subjects, $p<0.01$ (Tables 1,2).

\section{Discussion}

Fractal approaches grow in importance, year by year. Fractality, the geometric concept related to highly irregular shapes that originates from simple iterated function with noninteger, or fractional, dimensions and a property known as 

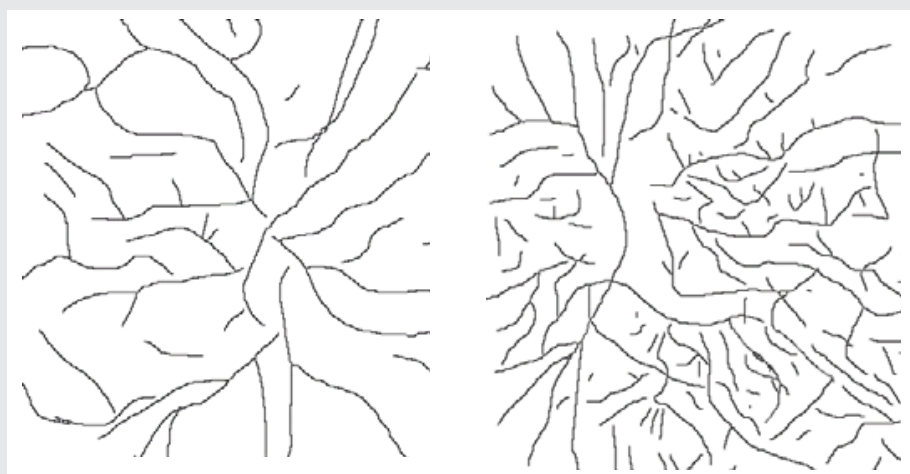

Figure 4: Trajectories of the optic nerve head microvascularity in a normal subject (left) and in a MS patient, affected eye (optic neuritis).

Table 1: Geometric complexity (D0) of the optic nerve head microvascularity in control subjects and in multiple sclerosis patients affected by optic neuritis.

\begin{tabular}{|c|c|c|c|}
\hline & eyes & DO(SD) & $p$ \\
\hline Control & 13 & $1.65(0.05)$ & \\
\hline
\end{tabular}

\begin{tabular}{|l|l|l|}
\hline MS patients, affected eye (ON) & 6 & $1.86(0.04)$
\end{tabular}

0.01

The values differed between the two groups $(p<0.01)$. Mean value (standard deviation).

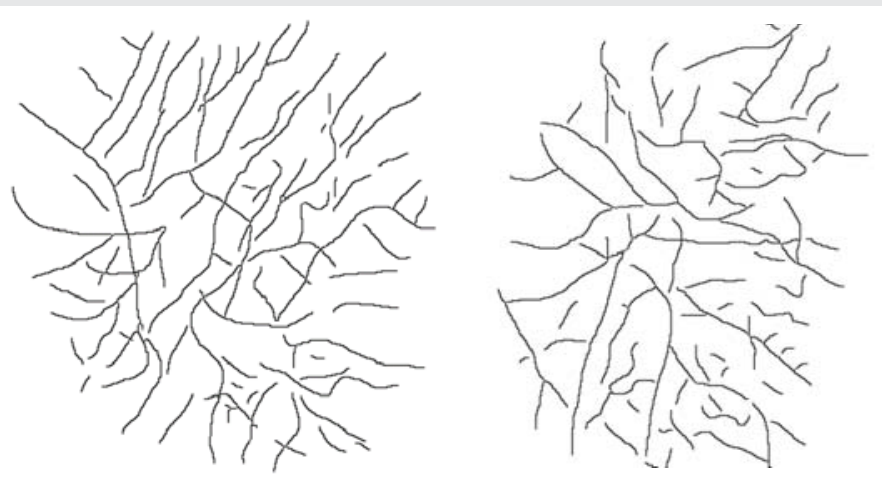

Figure 5: Trajectories of the optic nerve head microvascularity in a MS patient: affected eye (optic neuritis, left, $D 0=1.85$ ), unaffected eye (right, $D 0=1.83$ ).

Table 2: Geometric complexity (D0) of the optic nerve head microvascularity in multiple sclerosis patients affected by optic neuritis, affected eye vs. unaffected eye.

\begin{tabular}{|c|c|c|c|}
\hline & eyes & Do(SD) & $p$ \\
\hline MS patients, affected (ON) eye & 6 & $1.86(0.04)$ & \\
\hline MS patients, unaffected eye & 5 & $1.84(0.03)$ & n.s. \\
\hline
\end{tabular}

The values don't differ between the two groups $(p=n . s$.). Mean value (standard deviation). The geometric complexity of the unaffected eye in MS patients results higher than in normal control subjects (see Table 1, $p<0.01$ ).

self-similarity, give to the pathologist a powerful method to support diagnosis and prognosis of the patients [6-8].

Multiple sclerosis results from an immune-mediated inflammation and demyelination of axons. Lesions vary temporally and spatially, leading to the classic presentation of episodic neurologic symptoms. Among which, vision problems are potential early signs of MS. In effect, when MS affects the nerves in a person's eyes, it can cause optic neuritis. In effect, there is a known tendency for multiple sclerosis to develop in
ON patients, being the risk of progression to multiple sclerosis within two years of an acute attack of ON. It is important to establish differential diagnosis among the different causes of ON [3], in order to treat patients earlier.

Our results show that the mean geometric complexity of the microvascular pattern of the optic nerve head is higher in the eye affected by ON than in the eye of control subjects, according to our previous results $[16,17]$. Moreover, in these MS patients the eye not affected by ON, also, showed a higher geometric complexity of optic nerve head microvascularity than in the normal subjects, overlapping the data of the eye affected by ON. These data show a characteristic microvascular pattern of the optic nerve head in subjects affected by multiple sclerosis: if confirmed in a higher number of patients it can give us a tool to identify earlier subjects that can develop multiple sclerosis.

\section{References}

1. Milo R, Kahana E (2010) Multiple sclerosis: geoepidemiology, genetics and the environment. Autoimmun Rev Mar 5: A387-A394. Link: http://bit.ly/2S5v97t

2. Tsang BK, Macdonell R (2011) Multiple sclerosis- diagnosis, management and prognosis. Aust Fam Physician 40: 948-955. Link: http://bit.ly/374lwdt

3. (1991) The clinical profile of optic neuritis. Experience of the Optic Neuritis Treatment Trial. Optic Neuritis Study Group. Arch Ophthalmol 109: 1673-1678. Link: http://bit.ly/20ywyRW

4. Prasad S, Galetta SL (2010) Eye movement abnormalities in multiple sclerosis Neurol Clin 28: 641-655. Link: http://bit.ly/31wjuS8

5. Müri RM, Meienberg O (1985) The clinical spectrum of internuclear ophthalmoplegia in multiple sclerosis. Arch Neurol 42: 851-865. Link: http://bit.ly/387UPpw

6. Cross SS, Cotton DW (1992) The fractal dimension may be a usefu morphometric discriminant in histopathology. J Pathol 166: 409-411. Link: http://bit.ly/31wVi28

7. Losa GA, Nonnenmacher TF (1996) Self-similarity and fractal irregularity in pathologic tissues. Mod Pathol 9: 174-182. Link: http://bit.ly/2Sj0diQ

8. Heymans O, Fissette J, Vico P, Blacher S, Masset D, et al. (2000) Is fractal geometry useful in medicine and biomedical sciences?. Med Hypotheses 54 360-366. Link: http://bit.ly/2StkdzB

9. Daxer A (1992) Fractals and retinal vessels. Lancet 339: 618. Link http://bit.ly/3878uxg

10. Daxer A (1993) Characterization of the neovascularazitaion process in diabetic retinopathy by means of fractal geometry: diagnostic implications. Graefe's Arch Clin Exp Ophthalmol 231: 681-686. Link: http://bit.ly/2UvZ1eX

11. Daxer A (1995) Mechanism in retinal vasculogenesis: an analysis of the spatial branching site correlation. Curr Eye Res 14: 251-254. Link: http://bit.ly/2SoSpMP

12. Family F, Masters BR, Platt DE (1989) Fractal pattern formation in human retinal vessels. Physica D 38: 98-103. Link: https://go.aws/2vVo6pf

13. Cavallari M, Falco T, Frontali M, Romano S, Bagnato F, et al. (2011) Fracta analysis reveals reduced complexity of retinal vessels in CADASIL. PLos One 6: e19150. Link: http://bit.ly/2UxCPRq

14. Doubal FN, MacGillivray TJ, Patton N, Dhillon B, Dennis MS, et al. (2010) Fractal analysis of retinal vessels suggests that a distinct vasculopathy causes lacunar stroke. Neurology 74: 1102-1107. Link: http://bit.ly/31Cm75c 
15. Falconer K (1990) Fractal geometry: mathematical foundations and applications. Chichester John Wiley, NY, USA. Link:

16. Traversi C, Bianciardi G, Tasciotti A, Berni E, Nuti E, et al. (2008) Fractal analysis of fluoroangiographic patterns in anterior ischaemic optic neuropathy and optic neuritis: a pilot study. Clin Exp Ophtalmol 36: 323-328. Link: http://bit.ly/371WU5f
17. Bianciardi G, Latronico ME, Traversi C (2016) Mass Dimension Evaluation of the Optic Nerve Head Microvascularity in Non-Glaucomatous Optic Neuropathies. Int J Oral Craniofac Sci 2: 006-010. Link: http://bit.ly/2Uyr51k
Discover a bigger Impact and Visibility of your article publication with Peertechz Publications

\section{Highlights}

* Signatory publisher of ORCID

* Signatory Publisher of DORA (San Francisco Declaration on Research Assessment)

* Articles archived in worlds' renowned service providers such as Portico, CNKI, AGRIS, TDNet, Base (Bielefeld University Library), CrossRef, Scilit, J-Gate etc.

- Journals indexed in ICMJE, SHERPA/ROMEO, Google Scholar etc.

* OAI-PMH (Open Archives Initiative Protocol for Metadata Harvesting)

* Dedicated Editorial Board for every journal

* Accurate and rapid peer-review process

* Increased citations of published articles through promotions

* Reduced timeline for article publication

Submit your articles and experience a new surge in publication services (https://www.peertechz.com/submission).

Peertechz journals wishes everlasting success in your every endeavours.

Copyright: @ 2020 Bianciardi G, et al. This is an open-access article distributed under the terms of the Creative Commons Attribution License, which permits unrestricted use, distribution, and reproduction in any medium, provided the original author and source are credited. 